sind völlig analog. Zange 1 hat eine Fixierungsfläche von $2,25 \mathrm{~cm}^{2}$. Mit dieser viel kleineren Zange ist es möglich, die durch die Präparation irritierten Muskelpartien nahe dem Medianschnitt zu meiden und mehr lateral Gewebe zu entnehmen. Die Ergebnisse dieser Versuchsreihe zeigen einen weiteren Anstieg der energiereichen Phosphate ATP und $C P$ und einen deutlichen Abfall der glykolytischen Substrate. Bei der Beurteilung dieser Ergebnisse ist nur die unterschiedliche Zange zu berücksichtigen. Wenn mit dieser kleinen Zange Gewebe nahe dem Medianschnitt entnommen wurde, so entsprachen die erzielten Substratgehalte denen der Zange 2, so daß die Entnahme des Muskelgewebes weiter lateral und entfernt den am stärksten irritierten Muskelpartien für die niedrigen bzw. hohen Gehalte verantwortlich ist.

Bei der Betrachtung der Glucose-Werte in Tabelle 2 endlich ist $z u$ bedenken, daß Glucose nur in geringen Mengen intrazellulär vorkommt und der Hauptteil des gemessenen Gehaltes auf den extrazellulären Anteil entfällt. So ist der höhere Wert bei Benutzung einer größeren Zange (Spalte 2 der Tab. 2 im Vergleich zu Spalte 1) durch Reizung der Muskulatur und der noch höhere bei der HoHORST-Methode (Spalte 3 der Tab. 2) durch die Äthernarkose erklärbar. Äthernarkose führt zu einer beträchtlichen Hyperglykämie und außerdem $\mathrm{zu}$ einer Senkung des Glykogengehaltes. Letzteres ist auch bei Reizung der Muskulatur zu beobachten.

\title{
Literatur
}

1. Hohorst, H. J., Habilitationsschrift 1962, Marburg. - 2. Bücher, Th. und W. Rüssmann, Angew.. Chem. 75, 881 (1963). 3. Dubach, U. C., Klin. Wschr. 41, 157 (1963). - 4. Lowry, O. H., J. V. Passonneau, F. X. Hasselberger und D. W. Schulz, J. biol. Chemistry 239, 18 (1964). - 5. LowrY, O. H., J. V. PASSONNEAU und M. K. Rock, J. biol. Chemistry 236, 2756 (1961). 6. Lowry, O. H. und J. V. Passonneau, J. biol. Chemistry 239 , 31 (1964). - 7. Lowry, O. H., J. V. Passonneau, D. W. Schulz und M. Rock, J. biol. Chemistry 236, 2746 (1961). - 8. SerayDARIAN, K., W. F. H. M. MOMmaertS und A. WALlNer, J. biol. Chemistry 235, 2191 (1960). - 9. UdEnfriend, S., Fluorescence Assay in Biology and Medicine. Academic Press, London-New York (1962). - 10. Hohorst, H. J., F. H. KreUtZ und Th. Bücher, Biochem. Z. 332, 18 (1959). - 11. EмrICH, H. M. und R. K. H. Zwieber, Pflügers Arch. ges. Physiol. 290, 315 (1966).

Dr. R. Zwiebel MPI für Biophysik 6000 Frankfurt a. M. 70 Kennedy-Allee 70

\section{Die enzymatische Bestimmung von Glycerin im Blutserum und in Organextrakten}

\author{
Von H. J. Sertz und W. TaRnowski \\ Aus dem Pbysiologisch-Chemischen Institut der Universität Hamburg (Direktor: Prof. Dr. J. Kübnau)
}

(Eingegangen am 26. April 1968)

Die Leistungsfähigkeit der bisher angegebenen enzymatịschen Bestimmungsmethoden für Glycerin im Blutserum und in Organextrakten wird untersucht und kritisch diskutiert. Eine zuverlässige, schnelle und relativ billige Methode wird angegeben, die exakt reproduzierbare Werte liefert. Die starke Abhängigkeit der Serumglycerinwerte von der Art der Narkose wird demonstriert.

\section{The enzymic determination of glycerol in blood serum and in organ extracts}

The efficiency of available methods for the enzymic determination of glycerol in blood serum and organ extracts was studied and discussed critically. A reliable, rapid and relatively cheap method is reported, that yields exactly reproducible results. The strong dependance of the serum glycerol value on the nature of narcosis was demonstrated.

Die gebräuchlichen ${ }^{1}$ ) enzymatischen Bestimmungsmethoden für Glycerin basieren auf dessen Phosphorylierung zu Glycerin-1-phosphat durch ATP unter der katalytischen Wirkung des Enzyms Glycerokinase2):

Glycerin + ATP $\frac{\text { GK }}{\mathbf{M g}^{++}}$Glycerin-1-phosphat + ADP

1) Der von HAGEN und Hagen $(1,2)$ angegebene Nachweis mit Glycerindehydrogenase aus Aerobacter aerogenes hat sich nịcht durchgesetzt. Die Substrateichkurve der Methode ist keine Gerade. Daher müssen bei jeder Bestimmung eine Reibe von Eichstandards mitgeführt werden.

2) Folgende Abkürzungen und Bezeichnungen werden gebraucht: $\mathrm{PEP}=$ Phosphoenolpyruvat; TRA = Triäthanolamin; $\mathrm{GK}=$ Glycerokinase (EC 2.7.1.30); LDH = Lactatdehydrogenase (EC 1.1.1.27); $\mathrm{PK}=$ Pyruvatkinase (EC 2.7.1.40); $\mathrm{GDH}=$ Glycerin1-phosphatdehydrogenase (EC 1.1.1.8); Halothan $=1,1,1$,Trifluor-2-brom-2-chloräthan, Nembutal = Pentobarbital.
Diese Reaktion kann sichtbar gemacht werden, indem - nach dem Prinzip des gekoppelten optischen Testes von WARBURG - eines der Reaktionsprodukte nachgewiesen wird:

a) Glycerin-1-phosphat nach dem Vorschlag von Wieland (3) (vgl. auch (4), (5)):

$$
\begin{array}{r}
\text { Glycerin-1-phosphat } \\
+\mathrm{NAD}^{+}
\end{array} \quad \begin{array}{r}
\text { Dihydroxy-acetonphosphat } \\
+\mathrm{NADH}+\mathrm{H}^{+}
\end{array}
$$

Das Gleichgewicht dieser Reaktion liegt mit $K_{c}=$ $5,8 \cdot 10^{-12} \mathrm{Mol} / l$ (6) weit auf der linken Seite. Daher gelingt der quantitative Nachweis von Glycerin-1phosphat nur dann, wenn die Reaktionsprodukte aus dem Gleichgewicht entfernt werden: Dihydroxyacetonphosphat durch Hydrazin, Protonen durch ein al- 
kalisches Milieu. - Diese Methode wird im folgenden „Hydrazinmethode“ genannt.

b) Adenosindiphosphat nach dem Vorschlag von KREUTZ (7) (vgl. auch (8, 9)):

$$
\begin{array}{rll}
\text { ADP }+ \text { PEP } & \text { PE } & \text { ATP + Pyruvat } \\
\text { Pyruvat }+\mathrm{NADH}+\mathrm{H}^{+} \stackrel{\text { LDH }}{\longrightarrow} & \text { Lactat }+ \text { NAD }^{+}
\end{array}
$$

Das Gleichgewicht dieser gekoppelten Reaktionen liegt bei pH 7,6 weit auf der rechten Seite.

Beim Versuch, Glycerin im Blutserum und in Leberextrakten mit der Hydrazinmethode zu messen, fanden wir mitunter eine so schlechte Reproduzierbarkeit der Meßwerte, daß wir nicht umhin konnten, die Zuverlässigkeit der Methode noch einmal zu prüfen. Das Ergebnis war eine Modifikation der Methode von KreUtZ (7), mit der es nun möglich ist, Glycerin schnell und zuverlässig auch in hämolytischen Seren und in Organextrakten zu bestimmen.

\section{Die Hydrazinmethode}

Der Wert der Hydrazinmethode ist für biologisches Material begrenzt:

1. Es ist nicht möglich, mit steigenden Mengen von Leberextrakt eine Eichgerade zu erhalten (Abb. 1b), gleichgültig, ob man Ansatz I oder II (vgl. Legende zu $\mathrm{Abb} .1$ ) verwendet. Die Störung liegt offenbar beim

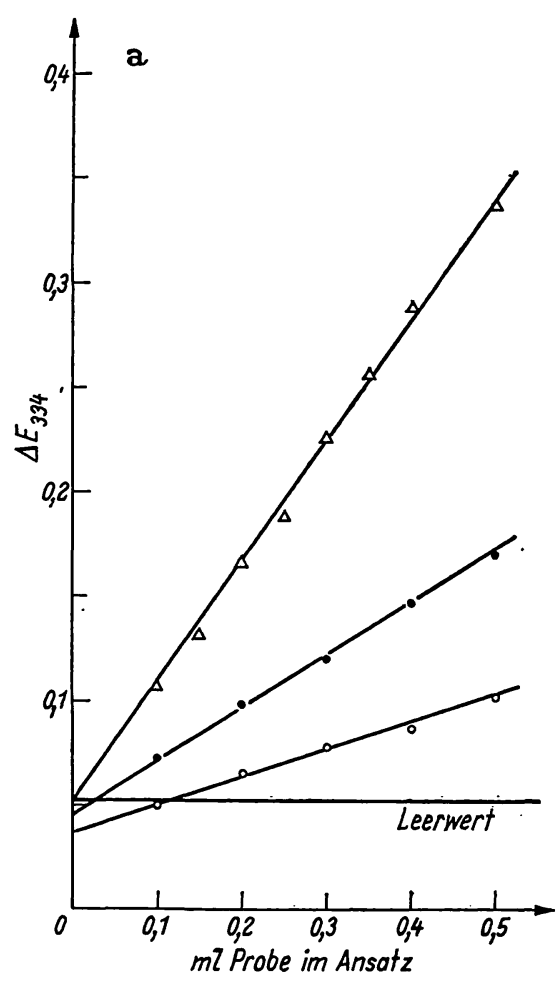

Abb. 1 a

Enzymatische Glycerinbestimmung

Ansatz I (nach (3)): $0,1-0,5 \mathrm{ml}$ neutraler Extrakt bzw. Glycerineichlösung, mit $\mathrm{H}_{2} \mathrm{O}$ auf $0,5 \mathrm{ml}$ Probevolumen ergänzt; $1,37 \mathrm{ml}$ Glycin ATP $(0,1 \mathrm{~m}) ; 0,05 \mathrm{ml} \mathrm{NAD}(0,02 \mathrm{M}) ; 0,02 \mathrm{ml} \mathrm{GDH}($ Boehringer, $10 \mathrm{mg} / \mathrm{ml})$; ATP $(0,1 \mathrm{M}) ; 0,02 \mathrm{ml} \mathrm{GK}($ Boehringer, $5 \mathrm{mg} / \mathrm{ml}) ;-\mathrm{d}=1 \mathrm{~cm}$

Glycerineichlösung: $2,5 \cdot 10^{-4} M(\Delta-\Delta)$

Leberhomogenat: 1 Tl. Leber +7 Tle. $\mathrm{KCl}(0,15 \mathrm{M}) / \mathrm{KHCO},(0,02 \mathrm{M})$ Leberhomogenat: 1 Tl. Leber $+7 \mathrm{Tle} . \mathrm{KCl}(0,15 \mathrm{M}) / \mathrm{KHCO}_{3}(0,02 \mathrm{M})$ $100000 \mathrm{~g} 20 \mathrm{Min}$. ultrazentrifugiert; Fallung mit dem gleichen Volumen $0,7 \mathrm{~N} \mathrm{HClO}$, i, neutralisiert mit $4 \mathrm{~N} \mathrm{KOH}$ (Titrisol Merck) (- Blutserum: Mit 0,7N HClO, im Verhältnis $1: 1(\mathrm{v} / \mathrm{v})$ enteiweißt und mit KOH (Titrisol Merck) neutralisiert ( $\circ-\circ)$. Alle Proben gegen Leerwert, Leerwert gegen Wasser gemessen

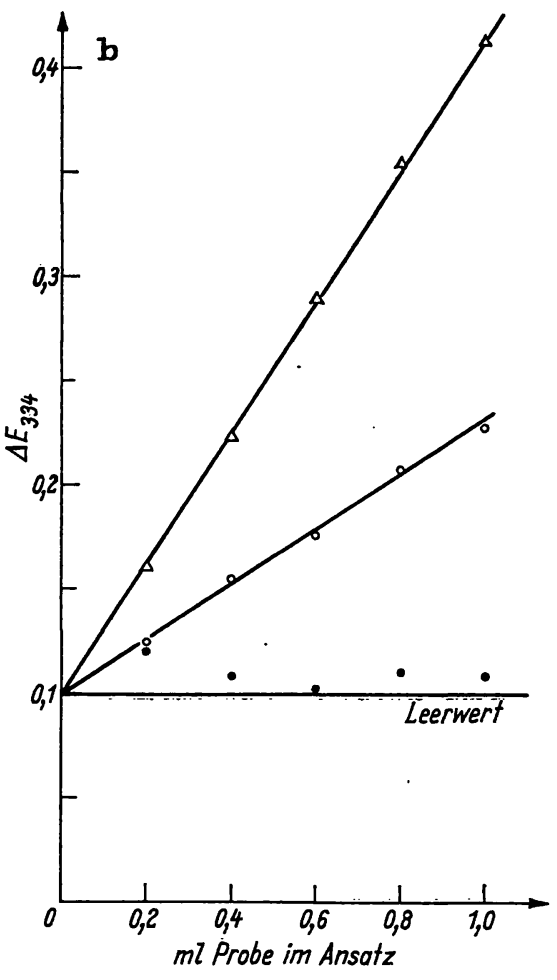

Abb. 1 b

Enzymatische Glycerinbestimmung

Ansatz II (nach (10)): 0,2-1,0 ml neutraler Extrakt bzw. Glycerinlösung, mit $\mathrm{H}_{2} \mathrm{O}$ auf $1,0 \mathrm{ml}$ Probevolumen ergänzt; $0,9 \mathrm{ml}$ Glycin (1M) /Hydrazinsulfat $(0,4 \mathrm{M}) / \mathrm{MgCl}_{2}(2 \mathrm{mM})$ - Puffer, $\mathrm{pH} 9,5 ; 0,025 \mathrm{ml}$ ATP $(0,1 \mathrm{M}) ; 0,1 \mathrm{ml} \mathrm{NAD}(0,05 \mathrm{M}) ; 0,02 \mathrm{ml}$ GDH (Boehringer, $10 \mathrm{mg} / \mathrm{ml})$; $0,02 \mathrm{ml}$ GK (Boehringer, $5 \mathrm{mg} / \mathrm{ml}$ ); $-\mathrm{d}=1 \mathrm{~cm}$ Glycerineichlösung: $1,25 \cdot 10^{-4}(\Delta-\Delta)$

Leberextrakt: 1 Tl Lber +4 Tle $07 \mathrm{~N}$ HClO 30 Sek mit dem Ultraturrax homogenisiert und neutralisiert mit KOH Titrisol Merck) und festem $\mathrm{KHCO}_{3}(\bullet-\bullet)$.

Übergang vom Glycerin zu Glycerin-1-phosphat und nicht bei der Indikatorreaktion, denn mit denselben Ansätzen erhält man für Glycerin-1-phosphat einwandfreie Eichgeraden. Es ist daher nicht möglich, in Leberextrakten Glycerin mit der Hydrazinmethode zu messen. 2. Mit steigenden Mengen eines Serumextraktes gibt die Hydrazinmethode eine einwandfreie Eichgerade. Verwendet man Ansatz I, dann schneidet diese die Ordinate aber nicht im Nullpunkt, sonder darunter (Abb. 1.a). Dieses Phänomen entsteht dadurch, daß in Ansätzen, die Extrakte biologischen Materials enthalten, die unspezifische Nachreaktion (der „Nachschleich") der Blindreaktion geringer ist als die der Proben. Der dadurch entstehende Fehler kann bis zu $25 \%$ betragen.

Größe und Divergenz des Nachschleichs erlauben es nicht, diese Reaktion als Endpunktreaktion zu behandeln (vgl. (11)).

Mißt man dieselben Proben mit Ansatz II, dann beobachtet man keine Störung des Tests (Abb. 1b).

3. Der Blindwert beträgt - unabhängig vom Ansatz $30-50 \%$ der Gesamtextinktion (Abb. 1). Seine Reproduzierbarkeit ist unbefriedigend (Für Ansatz I: $\pm 7 \%$; für Ansatz II: $\pm 2,5 \%$ ).

4. Die Hydrazinmethode ist $4-5 \mathrm{mal}$ so teuer wie die von uns ausgearbeitete Modifikation der Methode von KreUtz. 


\section{Die Methode von Kreutz}

Diese Methode hat den Vorteil, daß ihre Reaktionsfolge bei physiologischem $\mathrm{pH}$ spontan von links nach rechts und daher schnell abläuft. Ihr Ansatz enthält kein Hydrazin. Sie hat aber den Nachteil, daß die Proben nicht größere Mengen von ADP enthalten dürfen. Diese Voraussetzungen erfüllen weder Organextrakte noch Rattenblut, das fast immer hämolytisch ist. Wir haben die Schwierigkeit dadurch eliminiert, daß wir die Extrakte mit DEAE-Cellulose ausschüttelten. Mit

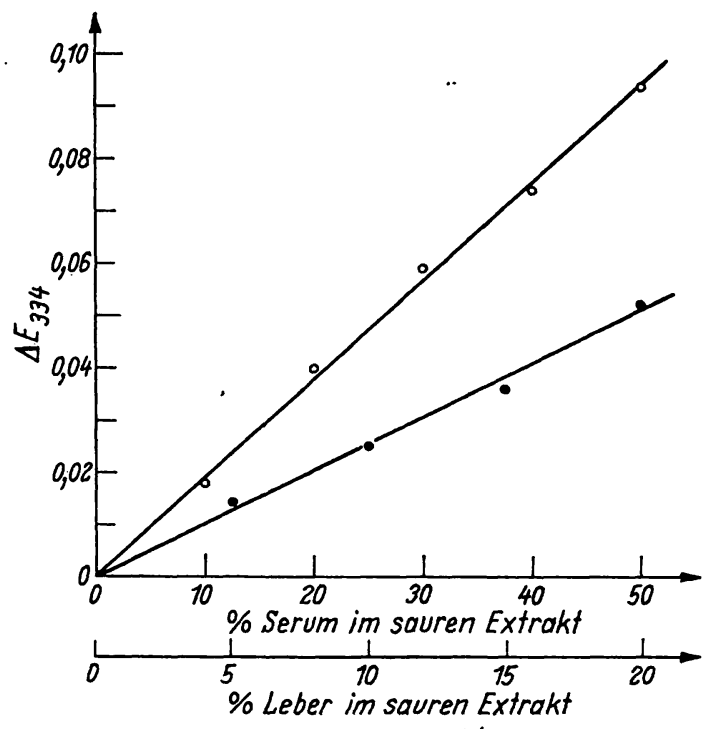

Abb. 2

Enzymatische Bestimmung von Glycerin nach KREUTz, modifiziert wie im Text beschrieben

$$
\text { - } 0 \text { in Serum in Leber }
$$

steigenden Mengen so behandelter Serum- und Leberextrakte erhält man exakte Eichgeraden durch den Nullpunkt (Abb. 2).

\section{Methodik}

\section{Unsere Methode umfaßt folgende Schritte:}

\section{Extraktion}

Blutserum wird im Verhältnis $1: 1(\nabla / v)$ mit $0,7 \mathrm{~N}$ eiskalter $\mathrm{HClO}_{4}$ enteiweißt. Leber wird mit maximal 4 Tln. derselben Säure 20 Sek. lang mit dem Ultraturrax homogenisiert. Eine Nachextraktion ist überflüssig. Eine Konzentration des Extrakts von $15 \%$ sollte nicht unterschritten werden, weil der Glyceringehalt des Blutes und der Organe sehr klein ist.

Beginnt die Extraktion spätestens 2 Min. nach dem Sistieren der Blutzirkulation in der Leber, dann ist eine Fixierung des Gewebes durch Gefrierstop (12) nicht nötig (Abb. 3). Nach dieser Zeit steigt die Glycerinkonzentration - wahrscheinlich durch Lipolyse von Organfett - langsam an.

\section{Neutralisation}

Das eiskalte Zentrifugat des sauren Extrakts wird mit vorgekühlter 4N Kalilauge und festem $\mathrm{KHCO}_{3}$ auf $\mathrm{pH} \mathrm{5-6}$ neutralisiert. Es muß sehr reine Lauge verwendet werden! Diese Bedingung erfüllen z. B. Titrisol-Kalilauge (Merck 9918) und Kaliumhydroxyd, Plätzchen für biochemische Zwecke (Merck 5026), nicht aber Kaliumhydroxyd, Plätzchen rein, pro analysi (Merck 5024) und reinst, DAB 6 (Merck 5032). Neutralisiert man 0,7 $\mathrm{N} \mathrm{HClO}_{4}$ mit Lösungen der letztgenannten Substanzen und setzt den Úberstand in einen der genannten enzymatischen Glycerinteste ein, dann kann man nach Zugabe von Glycerokinase eine regelrechte enzymatische Reaktion beobachten. Mit steigenden Mengen des Uberstandes erhält man eine Gerade. Das Phänomen wird nicht beobachtet, wenn man mit $\mathrm{KHCO}_{3}$ neutralisiert. Der Uberstand verhält sich mithin wic eine verdünnte Glycerinlösung. Die Neutralisation von Serumextrakten mit KOH p. a. (Merck 5024) erhöhte die Glycerinwerte um Faktor 3.

Das beschriebene Phänomen wurde kürzlich auch von Willms und SöLrNg (13) für eine andere Charge Kalilauge (Kaliumhydroxyd Plätzchen, reinst, pro analysi - Merck 5033) beobachtet. Die Autoren wiesen durch Papierchromatographie nach, daß die von ihnen untersuchte Lauge in dex Tat auf etwa 7000 Moleküle KOH 1 Molekül Glycerin als Verunreinigung enthält. Während der redaktionellen Bearbeitung dieses Manuskripts wurde das von WrLlms und SöliNg (13) und von uns beobachtete Phänomen von Schmid und DaHL (14) sowie - in einer Leserzuschrift an diese Zeitschrift - von KrEMER und TALKE (15) bestätigt.

\section{Adsorption von $A D P$}

$2 \mathrm{ml}$ des vom $\mathrm{KClO}_{4}$-Niederschlag dekantierten Überstandes werden in Zentrifugengläser mit $100 \mathrm{mg} \mathrm{DEAE-Cellulose} \mathrm{(OH-}$ Form) gegeben und geschüttelt (DEAE-Cellulose, Serva-Entwicklungslabor - Heidelberg, Nr. 45050; Kapazität 0,62 mVal/g,

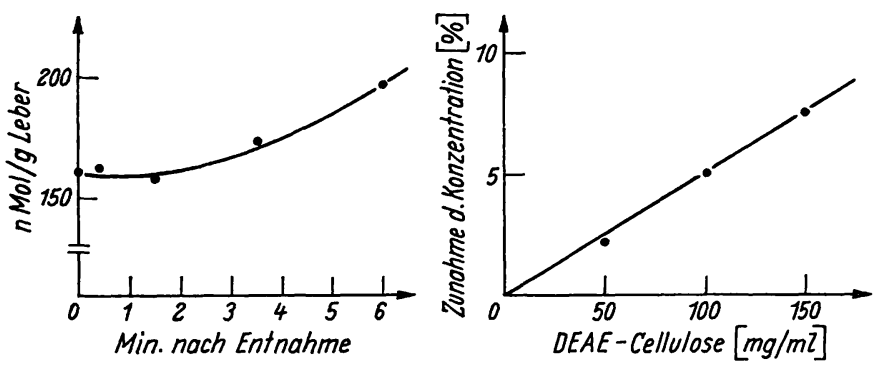

Abb. 3

Anstieg der Glycerinkonzentration in der Leber in Abhängigkeit von der Zeit nach Entnahme

Zeitpunkt 0 Min.: Gefrierstop

Abb. 4

Anstieg der Glycerinkonzentration in einer wäßr. Standardlösung in Abhängigkeit von der zugefügten Menge an DEAE-Cellulose

mit $0,5 \mathrm{~N} \mathrm{KOH}$ (höchster Reinheitl) aufgeschlemmt, gewaschen und getrocknet). Die Suspension soll im Ausschwingrotor zentrifugiert werden, weil der Cellulose-Niederschlag sehr locker ist. Eine unspezifische Adsorption von Glycerin findet nicht statt (Wiederfindung nach Zugabe zum neutralen Serumextrakt: $99,9 \pm 1,8 \%(n=4))$. Das Ausschütteln erhöht aber die Konzentration der Extrakte geringfügig (Abb. 4). Dieser Fehler ist für eine Charge DEAE-Cellulose konstant und kann daher rechnerisch korrigiert werden.

\section{Messung}

Testansat $z^{3}$ ):

Neutraler Extrakt

TRA-Puffer $(0,1 \mathrm{M})$, pH $7,6 / \mathrm{MgSO}_{4}(0,004 \mathrm{M})$

NADH $(0,006 \mathrm{M}) / \mathrm{ATP}(0,033 \mathrm{M}) / \operatorname{PEP}(0,11 \mathrm{M}) \quad 0,05 \mathrm{~m} /$

$\dot{\mathrm{L} D H}(2 \mathrm{mg} / \mathrm{m} l) / \mathrm{PK}(1 \mathrm{mg} / \mathrm{m} l) \quad 0,01 \mathrm{~m} l$

Start mit GK $(2 \mathrm{mg} / \mathrm{ml})$

Ansatzvolumen

$1,37 \mathrm{~m} l$

Messung: Photometer Eppendorf, $334 \mathrm{~nm}, \mathrm{~d}=1 \mathrm{~cm}$, gegen Leerküvette (s. u.).

Die Reaktion ist nach 7-10 Min. beendet. Ein regelmäßiger „Nachschleich“ muß extrapoliert werden. Dieser Nachschleich

3) Alle Substanzen von Boehringer \& Soehne, Mannheim. Dieser Ansatz entspricht den Lösungen der Boehringer-Testkombination TNAA 15989 (Glycerin und Neutralfett). 
beruht offenbar u. a. auf einer Verunreinigung der käuflichen GK durch Hexokinase (14). Man muß vor dem Start der Reaktion mit Glycerokinase einige Min. warten, bis die geringen ADPMengen, die als Beimengungen des ATP in den Test gelangen, reagiert haben.

Nach Ablauf der ADP-Reaktion bleibt bei biologischem Material in der Regel ein geringer Vorschleich bestehen, der die Genauigkeit der Messung aber nicht beeinträchtigt. Dieser Vorschleich kann allerdings in Leberextrakten unter bestimmten Bedingungen (z. B. bei Alloxandiabetes) so stark sein, daß eine exakte Messung dann nicht mehr möglich ist. In diesem Falle sollte anstelle der DEAE-Cellulose Aktivkohle $(20 \mathrm{mg} / \mathrm{ml}$ Extrakt) verwendet werden. Aktivkohle hat allerdings den Nachteil, auch Glycerin unspezifisch zu adsorbieren (Abb. 5). Diese Schwierigkeit kann

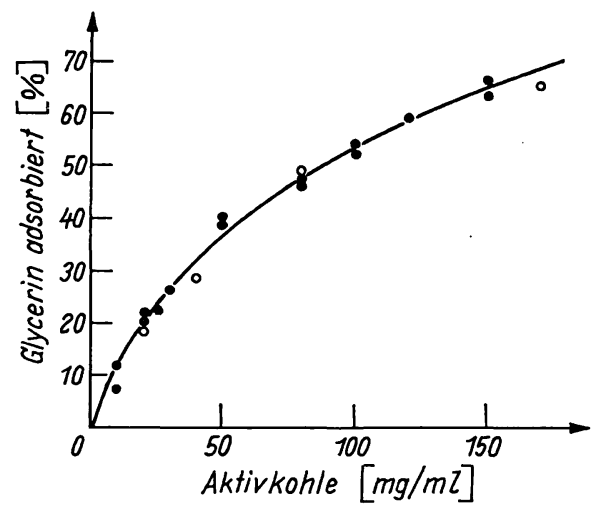

Abb. 5

Unspezifische Adsorption von Glycerin durch Aktivkohle aus einer wäßr. Standardlösung $(\bullet-\bullet-\bullet)$ und aus Leberextrakt (wie im
Text angegeben), der mit Glycerin angereichert wurde $(0 \div 0-0)$

wegen der geringen Glycerinkonzentrationen der Leber nicht durch Nachwaschen der Kohle beseitigt werden, wohl aber durch rechnerische Korrektur, da das Ausmaß der unspezifischen Adsorption für jede Charge der Aktivkohle konstant ist. Unter den angegebenen Bedingungen beträgt der Verlust 20\% (Abb. 5). Für jede Meßreihe muß eine Leerküvette mitlaufen, die anstelle des Extrakts Wasser enthält, da die Extinktion bei Zugabe von GK um 0,010-0,015 abnimmt.

Einfluß der Narkose auf den Serumglycerinspiegel

Eine Arbeit wie diese sollte an sich einige Normalwerte angeben. Aber zu einer solchen Angabe sind wir außer-

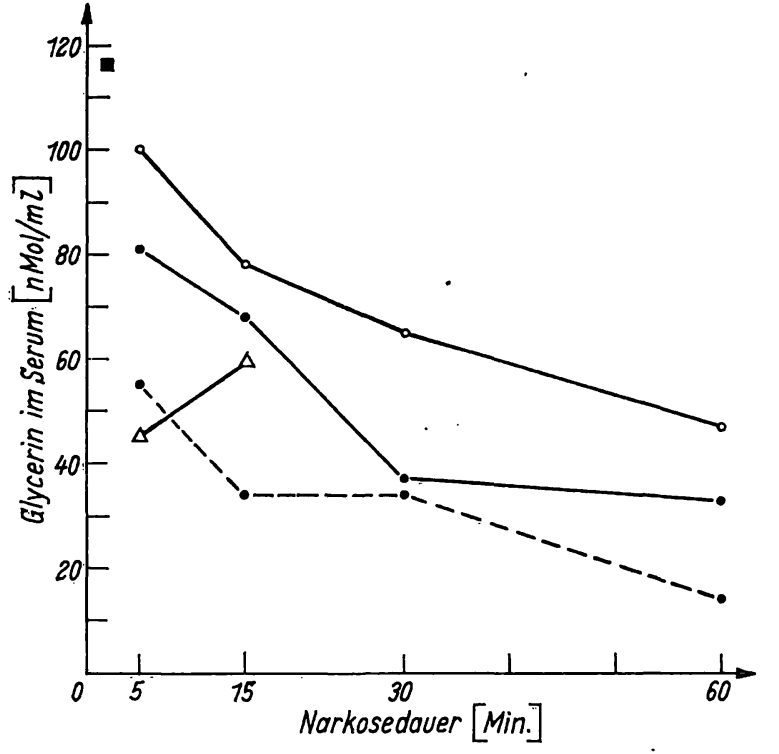

Abb. 6

Einfluß verschiedener Narkosearten auf den Serumglycerinspiegel von Rattenblut

Die Tiere wurden dekapitiert. $=$ Kopfschlag; $0-0=$ Halothan $(\sim 3 \%) / \mathrm{N}_{2} \mathrm{O}$-Sauerstoff $(1: 1)$; $16 \mathrm{mg} / 100 \mathrm{~g}$ Körpergewicht); $95 \% \mathrm{O}_{2} / 5 \% \mathrm{CO}_{2}(\mathrm{v} / \mathrm{v})$ - Atmosphäre $; \mathrm{A}=\mathrm{g}$ Korpergewicht)

stande. Die Serumglycerinspiegel in unseren Versuchen schwankten zwischen 14 und $117 \mathrm{nMol} / 100 \mathrm{~m} l$ Serum, je nachdem, welche Narkose vor dem Dekapitieren angewendet wurde und wie lange diese Narkose dauerte (Abb. 6). Die Abbildung zeigt, daß die Einleitung jeder Narkose zu einem Anstieg des Serumglycerins führt, der beim Kopfschlag maximal ist. Dauert die Narkose an, dann fallen die Glycerinspiegel kontinuierlich ab. Unsere Hoffnung, daß sich die Werte schließlich auf ein gemeinsames Niveau einpendeln würden, hat sich nicht erfüllt. Offensichtlich wird der Normalbereich bei den verschiedenen Narkosearten verschieden schnell durchlaufen und schließlich unterschritten.

Frl. M. SEEMANN danken wir für ihre zuverlässige technische Mitarbeit.

Der Deutschen Forschungsgemeinschaft danken wir für großzügige Unterstützung unserex Atbeit.

\section{Literatur}

1. HAGEN, J. H., Biochem. J. 82, 23 P (1962). - 2. HAGEN, J. H. und P. B. HAGEN, Canad. J. Biochem. Physiol. 40, 1129 (1962). 3. Wieland, O., Biochem. Z. 329, 313 (1957). - 4. Wreland, O., in Methoden der enzymatischen Analyse S. 211, Hrsg. H.-U. Bergmeyer, Verlag Chemie GmbH, Weinheim (1962). - 5. Boltralrk, J. J. und H. Nolt, Analytic. Biochem. 1, 269 (1960). - 6. HoHorsr, H. J., Dissertation, Universität Marburg (1960). - 7. KREUTZ, F. H., Klin. Wschr. 40, 362 (1962). - 8. GarLAND, P. B. und P. J. Randle, Nature London 196, 987 (1962). - 9. Panter, J. K., J. A. Hayashi und J. A. Watson, Arch. Biochem. Bio- physics 121, 404 (1967). - 10. HohoRst, H. J., in Methoden der enzymatischen Analyse S. 215, Hrsg. H.-U. Bergmeyer, Verlag Chemie GmbH, Weinheim (1962). - 11. Zöllner, N. und S. A. Warnock, Clin. chimica Acta Amsterdam 7, 607 (1962). - 12. Wollendberger, A., O. Ristau und G. Schofra, Pflügers Arch. Physiol. 270, 399 (1960). - 13. WILLms, B. und D. H. Söling, diese Z. 5, 276 (1967). - 14. SchmidT, F. H. und K. von DAHL, diese Z. 6, 156 (1968). - 15. Kremek, G. J. und H. TALKE, diese Z. 6, 223 (1968).

Doz. Dr. W. Tarnowski 2 Hamburg 20

Martinistraße 52

Physiolog.-Chem. Institut 